\title{
Prosociality in Palliative Nurses: Psychometric Evaluation of the Prosociality Scale
}

\begin{abstract}
Aim - The aim of this study was to examine the psychometric properties of a prosociality scale within the palliative nursing context, and then examine the impact of prosocial behaviour in relation to job and educational satisfaction among palliative nurses.

Methods-An online cross-sectional survey was conducted in 25 Italian palliative care centres, with a total of 107 nurses completing the prosociality scale by Caprara et al (2005). Exploratory and Confirmatory Factor Analyses were examined to evaluate a multi-dimensional model of prosociality.

Results-A three-factor solution with a second order factor fitted the data well. The three dimensions extracted were labelled as helping, empathy, and sharing. Participants reported high levels of prosociality. In addition, prosociality was positively associated with job and educational satisfaction.
\end{abstract}

Conclusions - The prosociality scale was valid and reliable when tested with palliative nurses. Although prosociality may be embedded in nurses' personalities, this quality should be actively promoted in order to expand and improve the culture and the ethics of nursing.

Keywords: prosociality, empathy, helping, palliative nurses, personality 


\section{Introduction}

According to Caprara and Bonino (2006), the term 'prosociality' refers to an individual disposition to engage in voluntary actions that result in positive effects for the benefit of another person. Prosociality is often conceived as an individual trait (Loke et al, 2011), and it can be argued that it is essential for nurses, especially for those providing palliative care, as they are required to establish valuable relationships with patients and families (Larkin, 2011). Actions such as sharing, caring, comforting, and supporting are not only all prosocial behaviours, but also inherent to nursing job description (Larkin, 2010). In order to address a care-pathway that is directed not only to the disease, but towards the holistic person (Watson, 1979), nurses need to engage in prosocial behaviours. Achieving such prosocial behaviour implies nurses holding ethical as well as moral values, which are also reflected and underpinned in the nursing profession in general and palliative care in particular.

Examples of prosocial values when applied to nursing are reported as resulting in: (a) mutual trust between patient and healthcare provider; (b) psychological proximity to patients; (c) empathy towards the patient's situation; (d) support for those who cannot manage on their own; (e) practical and relational knowledge; and (f) responsibility towards professional goals and obligations (Snellman and Gedda, 2012). Since the concept of prosociality can illustrate the nurse's attitude in moments of giving aid, it seems relevant to both the nursing literature and the palliative context, therefore it should be examined further. 
Nurses caring for patients during their palliative and end-of-life period may be more involved in showing prosocial behaviours towards patients and their families. Since palliative nursing is concerned mainly with promoting holism, dignity, and quality of life for the dying patient, palliative nurses are therefore required to care in accordance with principles espoused in the humanistic nursing theory (Wu and Volker, 2012), which include caring, empathy, and primacy of the nurse-patient relationship. In addition, palliative nurses are especially involved in communicating with patients and families about death-related issues. Dealing with such an emotion-laden and life limiting moments may require the development of advanced relational skills and ethical sensitivity (Weaver et al, 2008). Such approach helps to make a difference between 'being there' and 'being with' the dying patient (Haraldsdottir, 2011). Furthermore, nurses who chose to engage in end-of-life care were found to be primarily motivated by passion or a wish to pursue a social and human mission (Corli et al, 2006). Palliative nurses were also found to be more engaged in prosocial behaviours towards co-workers than nurses working in different clinical settings (Zaghini et al, 2015).

As the term 'prosociality' has rarely been used in nursing literature, scholars have focussed on the concepts of compassion (Schantz, 2007), altruism (Hamooleh et al, 2013), and empathy (Hojat, 2007). Compassion is intrinsic to the core of what it means to be a palliative 
nurse (Larkin, 2011), and it is described as a positive emotion associated with other elements such as empathy, fatigue, resilience, and love (Larkin, 2010). Altruism is an ethics-based aspect of palliative nursing, and it includes complete patient acceptance, supportive behaviour, and responsibility (Hamooleh et al, 2013). Empathy refers to the ability to carefully perceive the internal frame of reference of another person, as if one were the other, without ever losing the 'as if' condition (Rogers, 1975). Empathic people can understand others' concerns and communicate this understanding together with an intention to help (Hojat, 2007). Empathy in nurses is emphasised as human trait, professional state, and communication process (Kunyk and Olson, 2001). It is not only an essential component of the nurse-patient relation, but it is also an attribute of high-quality care delivery. Palliative nurses, indeed, seem to be more appreciated by patients and families if they show empathy (Spichiger, 2010).

Despite the importance of assessing and understanding prosocial behaviours in health care, particularly in palliative care, there is a paucity of reliable scales for measuring selfreported prosociality, as most of them focus merely on empathic tendencies (Hojat, 2007). In Italy, Caprara et al (2005) developed a scale to evaluate prosociality among adults. They conceived adult prosociality as those behaviours and feelings that reflect four types of actions: sharing, helping, taking care of, and feeling empathic concerns for others (Caprara et al, 2005). These actions, however, were hardly distinguishable from a psychometric perspective in a sample of general adults; thus, Caprara et al (2005) considered the adult prosociality scale as uni-dimensional. To examine the psychometric characteristics of the prosociality scale within 
the nursing professional context is important, as palliative nurses are the healthcare providers who spend more time with the patients and therefore engage in prosocial behaviours to a larger extent than professionals from other disciplines. Although all prosocial actions are likely to have a common source, identifying multiple dimensions of prosociality may help in nursing practice, education, and human resource management. For example, including prosocial dimensions in the nursing curriculum may help to humanise caring by promoting a personcentred approach (Fahrenwald et al, 2005).

In addition, we speculate that nurses with a higher prosociality may be more satisfied with their jobs and the education they received than those with low or no prosociality tendencies. The crucial point to emerge is that prosocial behaviours can be beneficial not only to the recipients but also to those who perform such actions. Therefore, the general idea is that prosociality generates satisfaction, which in turn increases prosocial attitudes, thereby leading to a virtuous circle that benefits both the patient and the health care professional.

Therefore, the aim of this study was to examine the psychometric properties of a prosociality scale within the palliative nursing context, and then examine the impact of prosocial behaviour in relation to job and educational satisfaction among palliative nurses.

\section{Methods}

Sample and Setting

A quantitative methodology was preferred to examine the psychometric properties of the prosociality scale within the palliative nursing context. An online cross-sectional survey, 
was conducted among 25 palliative care units: 18 (72\%) from the north, $2(8 \%)$ from the centre, and $5(20 \%)$ from the south of Italy. This was important to ensure the sample was not concentrated in one geographical area, thereby increasing representation as much as possible across Italy. The mean annual workload was of 495 patients per year $($ median $=241$; S.D. $=497$; range $=100-2,000)$, with costs covered by the National Health System for 22 centres $(88 \%)$. The mean number of nurses working in a centre was 14 (median $=10$; S.D. $=9$; range $=6-42$ ).

Participants were nurses working in palliative care in both hospice and at home, in public, non-profit or private organisations. The inclusion criteria for nurses to be eligible for participation in the study were:

- staff nurse or supervisor currently working in palliative care,

- being contactable via e-mail,

- ability to read and understand Italian,

- ability to use a computer connected to the internet.

Instruments

Prosociality was measured with the prosociality scale developed by Caprara et al (2005), a self-report tool to assess individual differences in adults' prosociality. Respondents were asked to indicate how often they engage in prosocial behaviours, such as 'trying to help others', in a Likert scale ranging from 1 (never) to 5 (always) (Caprara et al, 2005). Higher scores indicate higher prosociality. The prosociality scale was found to be uni-dimensional and showed high internal consistency (Cronbach's alpha $=0.91)$. 
Nurses were also asked about their job satisfaction. The subscale 'job in general' of the Job Descriptive Index (JDI) was used (Smith et al, 1969). It includes a list of 18 adjectives describing various aspects of the work experience. Participants are asked to indicate ' $Y$ ' if they agree, ' $\mathrm{N}$ ' if they disagree, and '?' if they are uncertain. Higher scores are consistent with greater job satisfaction, ranging $0-18$. In the present study, the internal consistency of the JDI sub-scale was 0.72 (Cronbach's alpha).

In addition, nurses were asked to rate the extent to which they felt satisfied with their professional education, using a scale ranging from 0 (not at all) to 10 (completely).

\section{Procedure}

Data were collected from August to November 2013. Palliative centres were selected on the basis of direct knowledge or availability of e-mail address on the web page of the Italian Federation of Palliative Care (FPC, 2013). A senior researcher first e-mailed a contact person for each palliative centre, in order to invite the nurses from the centre to participate in the study. After two working days, a researcher again e-mailed the contact person in order to forward the ethical approval for the study, together with a specific online form for collecting information about each palliative centre. The contact person agreed to participate in the study by contextually completing the online centre form. Then, the contact person received a link to the online nurse form, and was asked to forward it to all palliative nurses who met the study inclusion criteria. Thus, the contact person e-mailed the link to the palliative nurses working in that centre, asking them to participate in the study. The questionnaire was made with 
Computer Assisted Web Interviewing (CAWI) in Google Drive. Ethical approval for the study was obtained from the university with which the first author is affiliated.

\section{Statistical Analysis}

Means, standard deviations, and Pearson's correlations for all study variables were calculated. Since the validity of a scale (Furr, 2011), i.e. the degree to which scores can be interpreted in terms of a specific psychological construct, is directed by its dimensionality, which reflects the number and nature of variables assessed by its items, we examined the psychometric properties of the prosociality scale through Exploratory Factor Analysis (EFA) and Confirmatory Factor Analysis (CFA). EFA was used to preliminarily examine the dimensionality of the prosociality scale, with geomin oblique rotation (Comrey and Lee, 1992) To identify the number of factors to extract, multiple criteria were used, such as adequate fit indices (Hu and Bentler, 1999), the simplicity of the solution (factor loadings greater than 0.30 and no cross-loadings), interpretability of the factor structure, analysis of eigenvalues, and theoretical sense of the factor (Comrey and Lee, 1992). After having identified the best factor solution, CFA was used to cross-validate it. Due to non-normality of the item distribution, both EFA and CFA were performed using the maximum likelihood robust estimator (MLr) (Muthén and Muthén, 1998-2012). To evaluate the EFA and the CFA solutions, the following fit indices were considered: omnibus fit indices such as the Chi-square $\left(\chi^{2}\right)$, incremental fit indices such as the Comparative Fit Index (CFI; values $>0.90$ indicate a good fit) and the Tuker and Lewis Index (TLI; values $>0.90$ indicate a good fit), measures of fit in the sample such as the 
Standardised Root Mean Square Residual (SRMR; values $\leq 0.06$ indicate a good fit), and indices of approximation such as the Root Mean Square Error of Approximation (RMSEA; values $<0.06$ indicate a good fit) (Hu and Bentler, 1999). Statistical analyses were performed using Mplus 7.1(Muthén and Muthén, Los Angeles, CA, USA).

\section{Results}

Characteristics of the Sample

The sample included 107 palliative nurses (response rate $=31 \%$ ), mainly female $(84.9 \%$ ), with a mean age of 42 years (range $=24-63$ ), and their characteristics are shown in Table 1 . Most of them $(n=74 ; 70 \%)$ were married and achieved a university degree $(n=69 ; 64 \%)$, but only $14(13.5 \%)$ had a master's degree in palliative care. Participants mainly worked as staff nurses $(n=92 ; 87 \%)$ and exclusively in hospice $(n=63 ; 61 \%)$. Overall, the average work experience was 18 years, while it was 7 years in palliative care.

\section{Item Descriptive Statistics and EFA}

The items' descriptive statistics are shown in Table 2. All of the items were normally distributed (i.e. skewness and kurtosis indices $<|1|$ ), with the exception of items 1 and 9, which were negatively skewed. The mean for each item ranged between 3 and 4.6 (scale range $=1-5$ ), indicating high levels of prosocial behaviours.

The results of the EFA were consistent with a three-factor solution, which showed a better fit than one-factor or two-factor solutions while yielding a simple structure. The one- 
factor solution, indeed, yielded poor fit indices: $\chi^{2}(\mathrm{n}=107, \mathrm{df}=104)=195.9, \mathrm{p}<0.001 ; \mathrm{CFI}=$ 0.819; $\mathrm{TLI}=0.791 ; \mathrm{RMSEA}=0.091(90 \% \mathrm{CI}=0.071-0.110), \mathrm{p}=0.001 ; \mathrm{SRMR}=0.079$. Meanwhile, the three-factor model was found to have an adequate fit: $\chi^{2}(n=107, d f=75)=96.2, p=0.05$; $\mathrm{CFI}=0.958 ; \mathrm{TLI}=0.933 ; \mathrm{RMSEA}=0.051(90 \% \mathrm{CI}=0.000-0.079), \mathrm{p}=0.45 ; \mathrm{SRMR}=0.041$. The three factors were labelled as follows: (a) Factor 1: Helping, loaded by 6 items (20.4\% of the item total variance explained); (b) Factor 2: Sharing, loaded by 7 items (13.9\% of the item total variance explained); and (c) Factor 3: Empathy, loaded by 3 items (11.1\% of the item total variance explained) (Table 2). Overall, the three factors accounted for $45.4 \%$ of the item total variance. All of the primary factor loadings were adequate $(>0.30)$; they ranged from 0.33 for item 16 to 0.84 for item 13 . Each primary loading was at least two times greater than the secondary loading, with the exception of items 9,11 , and 16 , where the ratio between primary and secondary loading was respectively $1.7,1.4$, and 1.7 . Thus, simplicity of the factorial pattern was substantially supported.

\section{Reliability and CFA}

Cronbach's alpha coefficients were 0.83 for Helping, 0.75 for Sharing, and 0.76 for Empathy, indicating high reliability. The corrected item-total correlation coefficients were also adequate (>0.30), ranging between 0.40 and 0.69 for Helping, and between 0.53 and 0.62 for Empathy. However, with regard to Sharing, item 1 showed a poor item-total correlation coefficient $(r=0.287)$. In addition, this item was job-specific in the meaning (helping colleagues in their activities). It was, therefore, decided not to include item 1 in the Sharing score. 
Eliminating this item accounted for an increase in the Cronbach's alpha coefficient up to 0.76. We also decided to exclude item 16 because: (a) at EFA, it showed a greater factor loading with Sharing than with Empathy, although its meaning was related to empathy ('I immediately sense my friends' discomfort even when it is not directly communicated to me'); (b) the item-total correlation coefficient was not high $(\mathrm{r}=0.35)$; and $(\mathrm{c})$ the Cronbach's alpha coefficient did not change if the item was excluded.

The CFA was used to confirm the model found at the EFA (Table 2) but with items 1 and 16 excluded. In addition, since the correlations between the three factors were positive and strong, a second-order factor was specified in order to account for a comprehensive prosocial dimension, in line with Caprara et al (2005). This model fitted the data well: $\chi^{2}(\mathrm{n}=107, \mathrm{df}=74)$ $=102.17, \mathrm{p}=0.02, \mathrm{CFI}=0.949, \mathrm{TLI}=0.937, \mathrm{RMSEA}=0.060(\mathrm{CI} 95 \%=0.027-0.086), \mathrm{p}(\mathrm{RMSEA}<$ $0.05)=0.277, \mathrm{SRMR}=0.057$. All factor loadings were significant and higher than 0.40 (Fig.1). The second order factor was significantly loaded by the three first order factors, especially by Helping $(\lambda=0.96)$. Overall, the Cronbach's alpha was 0.88 .

\section{Scores and Correlations}

The mean score for Helping was $4.03(\mathrm{SD}=0.60$, range = 1.5-5), for Sharing was 3.96 (SD $=0.58$, range $=2-5)$, and for Empathy was $3.93(\mathrm{SD}=0.64$, range $=2.3-5)$. The mean for the total score was $3.99(\mathrm{SD}=0.50$, range $=2.2-5)$, indicating a high level of prosociality among participants. The correlations between factors were positive and significant: nurses with greater empathy engaged more in helping $(\mathrm{r}=0.51 ; \mathrm{p}<0.001)$ and sharing $(\mathrm{r}=0.44 ; \mathrm{p}<0.001)$, as well 
nurses who reported a higher level of helping behaviour were also more engaged in sharing $(r=$ $0.61 ; \mathrm{p}<0.001)$.

Participants reported a high satisfaction with their job (mean $=15.1, \mathrm{SD}=2.4)$ and with their professional education (mean $=7.5, \mathrm{SD}=2.1$ ). In particular, nurses who reported higher helping behaviours were more satisfied with their job $(\mathrm{r}=0.25 ; \mathrm{p}<0.01)$ and their professional education $(r=0.22 ; p<0.05)$. Also, nurses who reported higher sharing behaviours expressed greater job satisfaction $(\mathrm{r}=0.31 ; \mathrm{p}<0.001)$. The higher the level of satisfaction with the professional education, the more nurses were satisfied with their job $(r=0.22 ; p<0.05)$.

\section{Discussion}

The aim of this study was to examine the psychometric properties of the prosociality scale within the palliative nursing context, and then examine the level of this dimension in relation to job and educational satisfaction.

The prosociality scale by Caprara et al (2005) was shown to be valid and reliable in a sample of Italian palliative nurses. However, the four original types of actions - sharing, helping, taking care of, and feeling empathic with others-were not reflected into four psychometric dimensions, but into three: sharing, helping, and empathic behaviours. Nevertheless, the three-factor solution seems to include the major types of prosocial behaviours (Caprara and Bonino 2006). Sharing and feeling empathic with others did reflect into two unique dimensions. Helping and taking care of converged into one dimension. Thus, it is possible that those individuals who are engaged in caring activities for a work choice, such as nurses, refer to 
taking care of those who are in need as a way to help them (Wu and Volker, 2012). As these three dimensions were highly correlated with each other, a second-order factor was specified and found consistent with a comprehensive prosocial dimension, in line with the unidimensional solution by Caprara et al (2005).

Helping refers to voluntary aid or assistance provided to others who are in need. Helping decision-making is influenced by many factors, such as socio-cultural upbringing, moral values, social contexts, cognition, and personality traits (Penner et al, 2005). In addition, there are neural correlates of reasoning about helping decisions (Loke et al, 2011). The predisposition towards helping is a key element for palliative nurses, who aim to offer a support system to help patients and families cope during the illness (Larkin, 2010).

Sharing refers to the combined use of a resource or space, as the process of dividing and distributing. Sharing can actually mean giving something as a gift or providing information. Sharing is a basic component of human interaction, and is responsible for strengthening social ties. Sharing was also associated with the personality trait of agreeableness (Caprara et al, 2009). Agreeable individuals report positive and trustful perceptions of others, and they have not only a tendency to share their knowledge and abilities with others, but also to sacrifice their selfinterest in favour of other people (Caprara et al, 2012).

Lastly, Empathy was revealed as an integral part of the adult tendency to act prosocially, and not merely a correlate of it (Caprara et al, 2005), since an empathic individual feels sympathetic concerns that are the real reason for his prosocial behaviour (Caprara and Bonino, 2006). Empathy involves the ability to internally absorb another person's emotional condition. 
Empathic nurses are patient-oriented and focussed on patients' experiences while engaging in moral reasoning (Hoffman, 2001). The more nurses experience empathy for people in need, the more they feel responsible for them and have a desire to improve others' conditions (Paciello et al, 2013).

Participants reported high levels of prosociality, showing an individual disposition to engage in voluntary actions that result in positive effects for the benefit of another person. In addition, those who showed higher helping or sharing behaviours were more satisfied with their jobs as palliative nurses. Participants who reported to engage more frequently in helping behaviours believed that their professional education needs were met more than those with lower levels of helping behaviours. Thus, it is possible that education for nursing care elicits prosociality, or otherwise that prosocial individuals are more likely to choose nursing as a profession.

\section{Limits}

The findings of this study should be considered in light of some limitations. A first limit is the small sample size, mainly due to the low response rate to the online data collection. A larger number of participants would have enhanced the study validity, concerning both the statistical analysis (EFA and CFA) and the representativeness of the sample. A second limit is that nurses were not enrolled at random, but we followed a convenient sampling technique, with the possibility of self-selection of the participants. This may affect the results, since prosocial individuals may have been more likely to agree to take part in the study. In addition, 
prosociality is highly associated with social desirability bias. Another limit is that all of the items of the prosociality scale are positively worded, increasing the risk of response set bias.

\section{Conclusions}

The results of the present study suggest that the prosociality scale is valid and reliable also to use with palliative nurses. The high levels of prosocial tendencies among palliative nurses may translate to beneficial care outcomes for patients. In addition, nurses who are more engaged in prosocial behaviours can achieve a higher job satisfaction and also be more satisfied with their professional education. Although prosociality may be embedded in nurses' personality, it should be promoted in order to expand, in a positive way, the culture and the ethics of nursing. The practical relevance of promoting prosociality among nurses is the capacity to humanise caring across all health care spheres including palliative care settings. Anyway, since prosocial nurses are likely to be highly committed to care, they are in danger of developing stress leading to the burnout syndrome. Therefore, managers should ensure adequate monitoring and organisational support is offered to the nurses.

What remains unclear is whether nurses' prosocial behaviour remains constant over time. It is therefore recommended that future research examines whether prosociality changes over time and whether there are specific situations that may explain such changes. It is also important to understand whether and how prosocial behaviour is affected by educational programmes. 
Declaration of Conflicting Interests: The authors declared no potential conflicts of interest with respect to the research, authorship, and/or publication of this article. 


\section{References}

Caprara GV, Alessandri G, Di Giunta L, Panerai L, Eisenberg N (2009) The contribution of agreeableness and self-efficacy beliefs to prosociality. Eur J Pers 24(1): 36-55.

Caprara GV, Alessandri G, Eisenberg N (2012) Prosociality: the contribution of traits, values, and self-efficacy beliefs. J Pers Soc Psychol 102(6): 1289-1303.

Caprara GV, Bonino S (2006) Il comportamento prosociale. Aspetti individuali, familiari e sociali. Edizioni Erickson, Trento.

Caprara GV, Steca P, Zelli A, Capanna C (2005) A new scale for measuring adults' prosocialness. Eur J Psychol Assess 21(2): 77-89.

Comrey AL, Lee HB (1992) A first course in factor analysis. Lowrence Erlbaum associates Inc., Broadway, NJ.

Corli O, Marini MG, Andreoli G, et al (2006) Analisi del clima di lavoro nelle cure palliative. Progetto di indagine 2005. CIC Edizioni Internazionali, Trieste, IT.

Fahrenwald NL, Bassett SD, Tschetter L, Carson PP, White L, Winterboer VJ (2005) Teaching core nursing values. J Prof Nurs 21(1): 46-51.

FPC (2013) Federazione Cure Palliative Onlus. Hospice in Italia. Available at: http://www.fedcp.org/cure-palliative/hospice-in-italia.html.

Furr M (2011) Scale construction and psychometrics for social and personality psychology. SAGE Publications Ltd, London.

Hamooleh MM, Borimnejad L, Seyedfatemi N, Tahmasebi M (2013) Perception of Iranian nurses regarding ethics-based palliative care in cancer patients. J Med Ethics Hist Med 6: 1-8. 
Haraldsdottir E (2011) The constraints of the ordinary: 'being with' in the context of end-of-life nursing care. Int J Palliat Nurs 17(5): 245-250.

Hoffman ML (2001) Empathy and moral development: implications for caring and justice. Cambridge University Press, New York.

Hojat M (2007) Empathy in patient care: antecedents, development, measurement, and outcomes. Springer Science \& Business Media, New York.

Hu Lt, Bentler PM (1999) Cutoff criteria for fit indexes in covariance structure analysis: conventional criteria versus new alternatives. Structural Equation Modeling 6(1): 1-55.

Kunyk D, Olson JK (2001) Clarification of conceptualizations of empathy. J Adv Nurs 35(3): 317325.

Larkin PJ (2010) Listening to the still small voice: the role of palliative care nurses in addressing psychosocial issues at end of life. Prog Palliat Care 18(6): 335-340.

Larkin PJ (2011) Maintaining compassion in care planning. Int J Palliat Nurs 17(3): 107-107.

Loke IC, Evans AD, Lee K (2011) The neural correlates of reasoning about prosocial-helping decisions: an event-related brain potentials study. Brain Res 1369: 140-148.

Muthén L, Muthén B (1998-2012) Mplus user's guide. Muthén \& Muthén, Los Angeles, CA.

Paciello M, Fida R, Cerniglia L, Tramontano C, Cole E (2013) High cost helping scenario: the role of empathy, prosocial reasoning and moral disengagement on helping behavior. Pers Individ Dif 55(1): 3-7.

Penner LA, Dovidio JF, Piliavin JA, Schroeder DA (2005) Prosocial behavior: multilevel perspectives. Annu Rev Psychol 56: 365-392. 
Rogers CR (1975) Empathic: an unappreciated way of being. Couns Psychol 5(2): 2-10.

Schantz ML (2007) Compassion: a concept analysis. Nursing Forum 42(2): 48-55.

Smith PC, Kendall LM, Hulin CL (1969) The measurement of satisfaction in work and retirement. Rand McNally, Chicago.

Snellman I, Gedda KM (2012) The value ground of nursing. Nursing Ethics 19(6): 714-726.

Spichiger E (2010) Patients' and families' experience of their relationship with professional healthcare providers in hospital end-of-life care: an interpretive phenomenological study. J Hosp Palliat Nurs 12(3): 194-202.

Watson J (1979) Nursing: the philosophy and science of caring. Little Brown \& Co, Boston, MA.

Weaver K, Morse J, Mitcham C (2008) Ethical sensitivity in professional practice: concept analysis. J Adv Nurs 62(5): 607-618.

Wu H-L, Volker DL (2012) Humanistic Nursing Theory: application to hospice and palliative care. J Adv Nurs 68(2): 471-479.

Zaghini F, Biagioli V, Prandi C, Fida R, Sili A (2015) Nurses and organizational citizenship behavior: contribution to the Italian validation of the Podsakoff et al. scale. Med Lav 106(6): 460-471. 
Table 1. Socio-demographic and job characteristic of the sample $(\mathrm{N}=107)$

\begin{tabular}{lll}
\hline & $\mathrm{n}$ & $\%$ \\
\hline Sex & & \\
$\quad$ Male & 16 & 15.1 \\
$\quad$ Female & 90 & 84.9 \\
Age (mean, SD) & 41.6 & 9.6 \\
Marital status & & \\
$\quad$ Single & 22 & 20.8 \\
$\quad$ Married & 74 & 69.8 \\
$\quad$ Divorced & 8 & 7.5 \\
$\quad$ Widow & 2 & 1.9 \\
Education & & \\
$\quad$ Regional school & 35 & 33.7 \\
$\quad$ University (3 years) & 41 & 39.4 \\
$\quad$ Master in PC & 14 & 13.5 \\
$\quad$ University (>3 years) & 14 & 13.5 \\
Experience as nurse (mean, SD) & 17.9 & 11.0 \\
Experience as palliative nurse (mean, SD) & 6.8 & 5.2 \\
Workplace & & \\
$\quad$ Hospice & 63 & 61.2 \\
$\quad$ Home & 31 & 30.1 \\
$\quad$ Hospice and home & 9 & 8.7 \\
Job position & & \\
$\quad$ Staff nurse & 92 & 86.8 \\
$\quad$ Supervisor & 14 & 13.2 \\
\hline
\end{tabular}

Note: Work experience is measured in years 
Table 2. Statistics of the items of the prosociality scale and factor loadings for the EFA solution $(\mathrm{N}=107)$.

\begin{tabular}{|c|c|c|c|c|c|c|c|}
\hline Item & Mean & SD & Skew & Kurt & $\mathrm{F} 1$ & F2 & F3 \\
\hline 1. I am pleased to help my colleagues in their activities. & 4.57 & 0.66 & -2.07 & 7.03 & -.03 & $.41^{*}$ & -.14 \\
\hline 2. I share the things that I have with my friends. & 4.13 & 0.74 & -0.36 & -0.64 & -.07 & $.70^{*}$ & -.02 \\
\hline 3. I try to help others. & 4.33 & 0.71 & -0.73 & -0.07 & $.72^{*}$ & .20 & -.15 \\
\hline 4. I am available for volunteer activities to help those who are in need. & 3.03 & 1.08 & 0.04 & -0.58 & $.42^{*}$ & .10 & -.08 \\
\hline 5. I am empathic with those who are in need. & 4.12 & 0.76 & -0.47 & -0.35 & .22 & -.00 & $.59^{*}$ \\
\hline 6. I help immediately those who are in need. & 4.27 & 0.77 & -1.01 & 1.62 & $.71^{*}$ & -.03 & .11 \\
\hline 7. I do what I can to help others avoid getting into trouble. & 4.02 & 0.80 & -0.60 & 0.66 & $.56^{*}$ & .13 & .19 \\
\hline 8. I intensely feel what others feel. & 3.77 & 0.78 & -0.04 & -0.56 & .01 & .01 & $.80^{*}$ \\
\hline 9. I am willing to make my knowledge and abilities available to others. & 4.54 & 0.66 & -1.35 & 1.42 & .24 & $.40^{*}$ & .11 \\
\hline 10. I try to console those who are sad. & 4.27 & 0.73 & -0.77 & 0.26 & $.71^{*}$ & -.00 & .07 \\
\hline 11. I easily lend money or other things. & 3.13 & 1.07 & 0.01 & -0.45 & .14 & .36 & .25 \\
\hline 12. I easily put myself in the shoes of those who are in discomfort. & 3.91 & 0.80 & -0.52 & 0.66 & -.01 & .13 & $.58^{*}$ \\
\hline 13. I try to be close to and take care of those who are in need. & 4.29 & 0.71 & -0.65 & -0.19 & $.84^{*}$ & -.10 & .01 \\
\hline 14. I easily share with friends any good opportunity that comes to me. & 4.04 & 0.75 & -0.33 & -0.40 & .08 & $.73^{*}$ & .02 \\
\hline 15. I spend time with those friends who feel lonely. & 3.98 & 0.81 & -0.18 & -0.95 & .22 & $.52^{*}$ & -.00 \\
\hline $\begin{array}{l}\text { 16. I immediately sense my friends' discomfort even when it is not } \\
\text { directly communicated to me. }\end{array}$ & 4.13 & 0.70 & -0.19 & -0.94 & .01 & $.33^{*}$ & .20 \\
\hline
\end{tabular}

Note: EFA = Exploratory Factor Analysis; $S D=$ standard deviation; Skew = skewness; Kurt $=$ kurtosis; ${ }^{*} p<.05 ; F 1=$ Helping; F2=

Sharing; F3 = Empathy; primary factor loadings for each item are in bold. 
Figure 1. CFA model of the prosociality scale

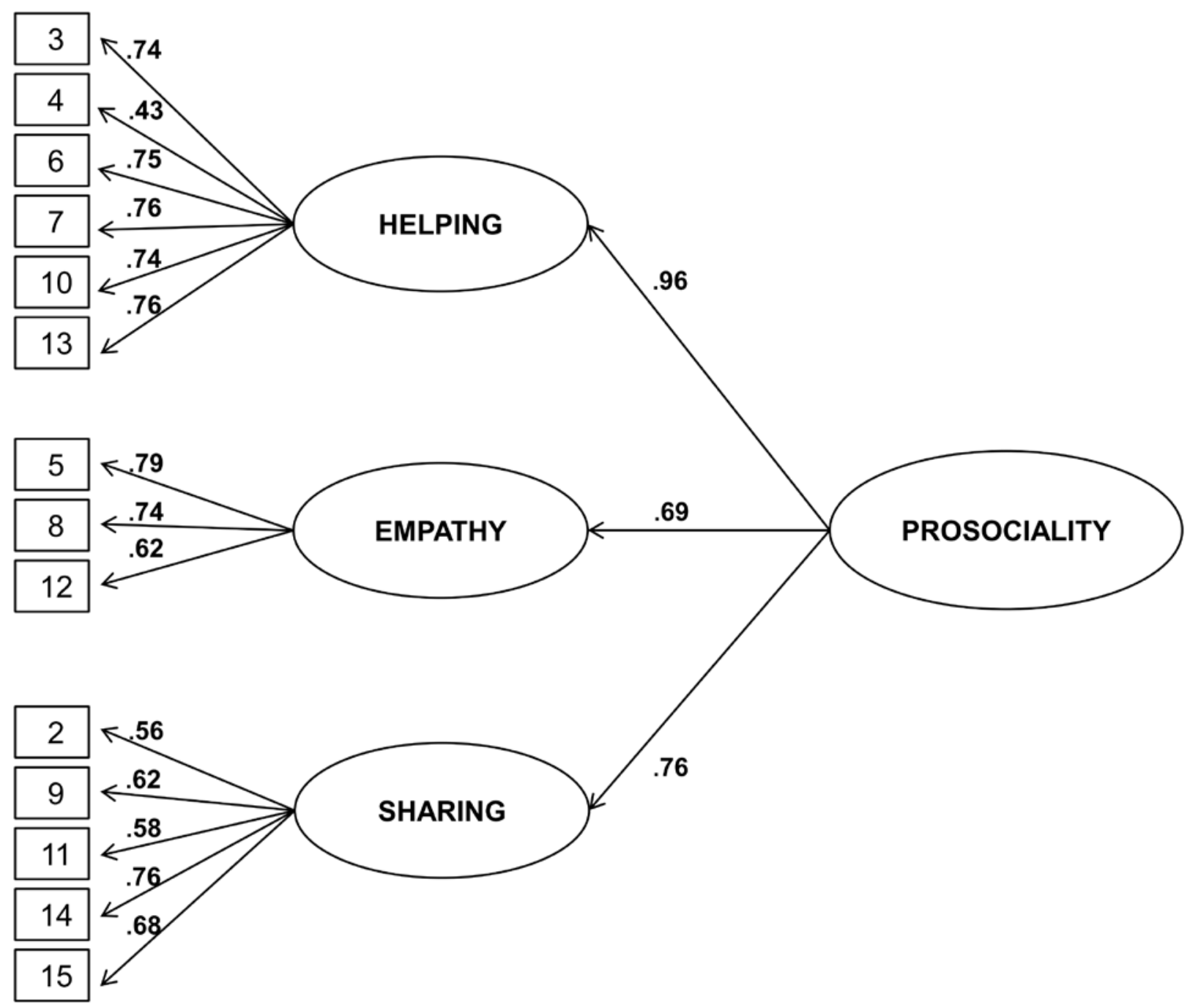


